\title{
SEMI-CONTINUOUS $G$-FRAMES IN HILBERT SPACES
}

\author{
ANIRUDHA PORIA
}

\begin{abstract}
In this paper, we introduce the concept of semi-continuous $g$-frames in Hilbert spaces. We first construct an example of semi-continuous $g$-frames using the Fourier transform of the Heisenberg group and study the structure of such frames. Then, as an application we provide some fundamental identities and inequalities for semi-continuous $g$-frames. Finally, we present a classical perturbation result and prove that semi-continuous $g$-frames are stable under small perturbations.
\end{abstract}

Вводиться поняття напівнеперервного $g$-фрейму в гільбертовім просторі. Спочатку будується приклад напівнеперервного $g$-фрейму, який спирається на перетворення Фур'є на групі Гейзенберга. Досліджується структура таких фреймів. Як застосування, отримані деякі фундаментальні тотожності та нерівності для напівнеперервних $g$-фреймів. Нарешті, доведено теорему про збурення: напівнеперервні $g$-фрейми стійкі відносно малих збурень.

\section{INTRODUCTION}

Discrete and continuous frames arise in many applications in mathematics and, in particular, they play important roles in scientific computations and digital signal processing. The concept of a frame in Hilbert spaces has been introduced in 1952 by Duffin and Schaeffer [13], in the context of nonharmonic Fourier series (see [29]). After the work of Daubechies et al. [11] frame theory got considerable attention outside signal processing and began to be more broadly studied (see $[8,22])$. A frame for a Hilbert space is a redundant set of vectors in Hilbert space which provides non-unique representations of vectors in terms of frame elements. The redundancy and flexibility offered by frames has spurred their application in several areas of mathematics, physics, and engineering such as wavelet theory, sampling theory, signal processing, image processing, coding theory and many other well known fields. Applications of frames, especially in the last decade, motivated researchers to find some generalization of frames like continuous frames [1, 23], $g$-frames [27], Hilbert-Schmidt frames [25, 26], $K$-frames [19, 20] and etc. Our main purpose in this paper is to study a generalization of frames, namely semi-continuous $g$-frames, which are natural generalizations of $g$-frames and continuous $g$-frames. We investigate the structure of semi-continuous $g$-frames and establish some identities and inequalities of these frames. Also, we present a perturbation result and discuss the stability of the perturbation of a semi-continuous $g$-frame.

Throughout this paper, $\mathcal{H}$ and $\mathcal{K}$ are two Hilbert spaces; $J$ is a countable index set; $(\mathcal{X}, \mu)$ is a measure space with positive measure $\mu ;\left\{\mathcal{K}_{x}\right\}_{x \in \mathcal{X}}$ is a family of closed subspaces of $\mathcal{K} ; \mathcal{L}\left(\mathcal{H}, \mathcal{K}_{x}\right)$ is the collection of all bounded linear operators from $\mathcal{H}$ into $\mathcal{K}_{x} ;$ if $\mathcal{K}_{x}=\mathcal{H}$ for any $x \in \mathcal{X}$, we denote $\mathcal{L}\left(\mathcal{H}, \mathcal{K}_{x}\right)$ by $\mathcal{L}(\mathcal{H})$.

We recall that a family $\left\{f_{j}\right\}_{j \in J}$ in $\mathcal{H}$ is called a (discrete) frame for $\mathcal{H}$, if there exist constants $0<A \leq B<\infty$ such that

$$
A\|f\|^{2} \leq \sum_{j \in J}\left|\left\langle f, f_{j}\right\rangle\right|^{2} \leq B\|f\|^{2}, \quad \forall f \in \mathcal{H} .
$$

2020 Mathematics Subject Classification. Primary 42C15; Secondary 47B38, 42C40.

Keywords. $g$-frames; continuous $g$-frames; semi-continuous $g$-frames; perturbation; frame identity; stability. 
The concept of the discrete frame was generalized to continuous frame by Kaiser [23] and independently by Ali et al. [1]. A family of vectors $\left\{\psi_{x}\right\}_{x \in \mathcal{X}} \subseteq \mathcal{H}$ is called a continuous frame for $\mathcal{H}$ with respect to $(\mathcal{X}, \mu)$, if $\left\{\psi_{x}\right\}_{x \in \mathcal{X}}$ is weakly-measurable, i.e., for any $f \in \mathcal{H}, x \rightarrow\left\langle f, \psi_{x}\right\rangle$ is a measurable function on $\mathcal{X}$, and if there exist two constants $A, B>0$ such that

$$
A\|f\|^{2} \leq \int_{\mathcal{X}}\left|\left\langle f, \psi_{x}\right\rangle\right|^{2} d \mu(x) \leq B\|f\|^{2}, \quad \forall f \in \mathcal{H} .
$$

Continuous frames have been widely applied in continuous wavelets transform [2] and the short-time Fourier transform [22]. We refer to $[3,16,17]$ for more details on continuous frames.

The notion of a discrete frame was extended to $g$-frame by Sun [27], which generalized all the existing frames such as bounded quasi-projectors [15], frames of subspaces [7], pseudo-frames [24], oblique frames [9], etc. $G$-frames are natural generalizations of frames as members of a Hilbert space to bounded linear operators. Let $\left\{\mathcal{K}_{j}: j \in J\right\} \subset \mathcal{K}$ be a sequence of Hilbert spaces. A family $\left\{\Lambda_{j} \in \mathcal{L}\left(\mathcal{H}, \mathcal{K}_{j}\right): j \in J\right\}$ is called a $g$-frame, for $\mathcal{H}$ with respect to $\left\{\mathcal{K}_{j}: j \in J\right\}$ if there are two constants $A, B>0$ such that

$$
A\|f\|^{2} \leq \sum_{j \in J}\left\|\Lambda_{j}(f)\right\|^{2} \leq B\|f\|^{2}, \quad \forall f \in \mathcal{H} .
$$

The continuous $g$-frames were proposed by Dehghan and Hasankhani Fard in [12], which are an extension of $g$-frames and continuous frames. A family $\left\{\Lambda_{x} \in \mathcal{L}\left(\mathcal{H}, \mathcal{K}_{x}\right)\right.$ : $x \in \mathcal{X}\}$ is called a continuous $g$-frame for $\mathcal{H}$ with respect to $(\mathcal{X}, \mu)$, if $\left\{\Lambda_{x}: x \in \mathcal{X}\right\}$ is weakly-measurable, i.e., for any $f \in \mathcal{H}, x \rightarrow \Lambda_{x}(f)$ is a measurable function on $\mathcal{X}$, and if there exist two constants $A, B>0$ such that

$$
A\|f\|^{2} \leq \int_{\mathcal{X}}\left\|\Lambda_{x}(f)\right\|^{2} d \mu(x) \leq B\|f\|^{2}, \quad \forall f \in \mathcal{H} .
$$

Notice that if $\mathcal{X}$ is a countable set and $\mu$ is a counting measure, then the continuous $g$-frame is just the $g$-frame. By the Riesz representation theorem, for any $\Lambda \in \mathcal{L}(\mathcal{H}, \mathbb{C})$, there exist a $h \in \mathcal{H}$, such that $\Lambda(f)=\langle f, h\rangle$ for all $f \in \mathcal{H}$. Hence, if $\mathcal{K}_{x}=\mathbb{C}$ for any $x \in \mathcal{X}$, then the continuous $g$-frame is equivalent to the continuous frame.

This paper is organized as follows. After the introduction, in Section 2, we introduce the semi-continuous $g$-frames in Hilbert spaces and construct an example using the Fourier transform of the Heisenberg group. Then we study the structure of semicontinuous $g$-frames using shift-invariant spaces. In Section 3, we first list some fundamental identities and inequalities of discrete frames just for the contrast to the main results of this section. Then we derive some important identities and inequalities of semicontinuous $g$-frames. Finally, in Section 4, we present a classical perturbation result and prove that semi-continuous $g$-frames are stable under small perturbations.

\section{Semi-Continuous G-Frames}

Let $\left\{\mathcal{K}_{x, j}: x \in \mathcal{X}, j \in J\right\} \subset \mathcal{K}$ be a family of Hilbert spaces.

Definition 2.1. A family $\left\{\Lambda_{x, j} \in \mathcal{L}\left(\mathcal{H}, \mathcal{K}_{x, j}\right): x \in \mathcal{X}, j \in J\right\}$ is called a semi-continuous $g$-frame for $\mathcal{H}$ with respect to $(\mathcal{X}, \mu)$, if $\left\{\Lambda_{x, j}: x \in \mathcal{X}, j \in J\right\}$ is weakly-measurable, i.e., for any $f \in \mathcal{H}$ and any $j \in J$, the function $x \rightarrow \Lambda_{x, j}(f)$ is measurable on $\mathcal{X}$, and if there exist two constants $A, B>0$ such that

$$
A\|f\|^{2} \leq \int_{\mathcal{X}} \sum_{j \in J}\left\|\Lambda_{x, j}(f)\right\|^{2} d \mu(x) \leq B\|f\|^{2}, \quad \forall f \in \mathcal{H} .
$$

If only the right-hand inequality of (2.1) is satisfied, we call $\left\{\Lambda_{x, j}: x \in \mathcal{X}, j \in J\right\}$ the semi-continuous $g$-Bessel sequence for $\mathcal{H}$ with respect to $(\mathcal{X}, \mu)$ with Bessel bound $B$. 
Remark 2.2. If $0<\mu(\mathcal{X})<\infty$, and for any fixed $x \in \mathcal{X}$, the family $\left\{\Lambda_{x, j}: j \in J\right\}$ is a $g$-frame for $\mathcal{H}$ with respect to $\left\{\mathcal{K}_{x, j}: j \in J\right\}$, then $\left\{\Lambda_{x, j}: x \in \mathcal{X}, j \in J\right\}$ is a semicontinuous $g$-frame for $\mathcal{H}$ with respect to $(\mathcal{X}, \mu)$. Moreover if $|J|<\infty$, and for any fixed $j \in J$, the family $\left\{\Lambda_{x, j}: x \in \mathcal{X}\right\}$ is a continuous $g$-frame for $\mathcal{H}$, then $\left\{\Lambda_{x, j}: x \in \mathcal{X}, j \in J\right\}$ is a semi-continuous $g$-frame for $\mathcal{H}$ with respect to $(\mathcal{X}, \mu)$.

In the following, we shall construct an example of such frames using the Fourier transform of the Heisenberg group.

2.1. Heisenberg Group. The Heisenberg group $\mathbb{H}$ is a Lie group whose underlying manifold is $\mathbb{R}^{3}$. We denote points in $\mathbb{H}$ by $(p, q, t)$ with $p, q, t \in \mathbb{R}$, and define the group operation by

$$
\left(p_{1}, q_{1}, t_{1}\right)\left(p_{2}, q_{2}, t_{2}\right)=\left(p_{1}+p_{2}, q_{1}+q_{2}, t_{1}+t_{2}+\frac{1}{2}\left(p_{1} q_{2}-q_{1} p_{2}\right)\right) .
$$

It is easy to verify that this is a group operation, with the origin $0=(0,0,0)$ as the identity element. Notice that the inverse of $(p, q, t)$ is given by $(-p,-q,-t)$. The Haar measure on the group $\mathbb{H}=\mathbb{R}^{3}$ is the usual Lebesgue measure.

The irreducible representations of the Heisenberg group has been identified by all non-zero elements in $\mathbb{R}^{*}(=\mathbb{R} \backslash\{0\})$ (see [14]). Indeed, for any $\lambda \in \mathbb{R}^{*}$, the associated irreducible representation $\rho_{\lambda}$ of $\mathbb{H}$ is equivalent to Schrödinger representation into the class of unitary operators on $L^{2}(\mathbb{R})$, such that for any $(p, q, t) \in \mathbb{H}$ and $f \in L^{2}(\mathbb{R})$, the operator $\rho_{\lambda}(p, q, t)$ is defined by

$$
\rho_{\lambda}(p, q, t) f(x)=e^{i \lambda t} e^{i \lambda\left(p x+\frac{1}{2}(p q)\right)} f(x+q) .
$$

It is easy to see that $\rho_{\lambda}(p, q, t)$ is a unitary operator satisfying the homomorphism property:

$$
\rho_{\lambda}\left(\left(p_{1}, q_{1}, t_{1}\right)\left(p_{2}, q_{2}, t_{2}\right)\right)=\rho_{\lambda}\left(p_{1}, q_{1}, t_{1}\right) \rho_{\lambda}\left(p_{2}, q_{2}, t_{2}\right) .
$$

Thus each $\rho_{\lambda}$ is a strongly continuous unitary representation of $\mathbb{H}$. By Stone and von Neumann theorem ([14]), $\left\{\rho_{\lambda}: \lambda \in \mathbb{R}^{*}\right\}$ are all the infinite dimensional irreducible unitary representations of $\mathbb{H}$, whose set has non-zero Plancherel measure. The measure $|\lambda| d \lambda$ is the Plancherel measure on the dual space $\widehat{\mathbb{H}}\left(\cong \mathbb{R}^{*}\right)$ of $\mathbb{H}$, and $d \lambda$ is the Lebesgue measure on $\mathbb{R}^{*}$. For $\varphi \in L^{2}(\mathbb{H})$ and $\lambda \in \mathbb{R}^{*}$, we denote $\widehat{\varphi}(\lambda)$ the operator-valued Fourier transform of $\varphi$ at a given irreducible representation $\rho_{\lambda}$, which is defined by

$$
\widehat{\varphi}(\lambda)=\int_{\mathbb{H}} \varphi(x) \rho_{\lambda}(x) d x .
$$

The operator $\widehat{\varphi}(\lambda)$ is a unitary map on $L^{2}(\mathbb{R})$ into $L^{2}(\mathbb{R})$, such that for any $f \in L^{2}(\mathbb{R})$

$$
\widehat{\varphi}(\lambda) f(y)=\int_{\mathbb{H}} \varphi(x) \rho_{\lambda}(x) f(y) d x .
$$

Therefore $\widehat{\varphi}(\lambda)$ belongs to $L^{2}(\mathbb{R}) \otimes L^{2}(\mathbb{R})$. If $\varphi \in L^{2}(\mathbb{H}), \widehat{\varphi}(\lambda)$ is actually a Hilbert-Schmidt operator on $L^{2}(\mathbb{R})$ and from the Plancherel theorem we have

$$
\int_{\mathbb{H}}|\varphi(x)|^{2} d x=\int_{\mathbb{R}^{*}}\|\widehat{\varphi}(\lambda)\|_{H . S .}^{2}|\lambda| d \lambda,
$$

the norm $\|\cdot\|_{H . S .}$ denotes the Hilbert-Schmidt norm in $L^{2}(\mathbb{R}) \otimes L^{2}(\mathbb{R})$. The proof of the Plancherel theorem for the Heisenberg group can be found in [21], and for more general groups, see [14].

To construct our example of semi-continuous $g$-frames, we shall define another unitary operator as follows. 
Let $\Pi:=[0,1]$ and $\mathfrak{L}:=\ell^{2}\left(\mathbb{Z}, L^{2}(\mathbb{R}) \otimes L^{2}(\mathbb{R})\right)$ be the Hilbert space of all sequences with values in the space $L^{2}(\mathbb{R}) \otimes L^{2}(\mathbb{R})$, i.e.,

$$
\mathfrak{L}=\left\{\left\{a_{n}\right\}_{n \in \mathbb{Z}}: a_{n} \in L^{2}(\mathbb{R}) \otimes L^{2}(\mathbb{R}) \text { and } \sum_{n \in \mathbb{Z}}\left\|a_{n}\right\|_{H . S .}^{2}<\infty\right\} .
$$

Lemma 2.3. For any $\sigma \in \Pi$, let $T_{\sigma}: L^{2}(\mathbb{H}) \rightarrow \mathfrak{L}$ given by $T_{\sigma} f(j)=|\sigma+j|^{\frac{1}{2}} \widehat{f}(\sigma+j)$. Then $T_{\sigma}$ is well-defined and $\sum_{j \in \mathbb{Z}}|\sigma+j|\|\widehat{f}(\sigma+j)\|_{H . S .}^{2}<\infty$.

Proof. Let $f \in L^{2}(\mathbb{H})$. Using Plancherel theorem and an application of periodization method, we obtain

$$
\begin{aligned}
\|f\|_{L^{2}(\mathbb{H})}^{2}=\int_{\mathbb{R}^{*}}\|\widehat{f}(\lambda)\|_{H . S .}^{2}|\lambda| d \lambda & =\int_{\sigma \in \Pi} \sum_{j \in \mathbb{Z}}|\sigma+j|\|\widehat{f}(\sigma+j)\|_{H . S .}^{2} d \sigma \\
& =\int_{\sigma \in \Pi} \sum_{j \in \mathbb{Z}}\left\|T_{\sigma} f(j)\right\|_{H . S .}^{2} d \sigma .
\end{aligned}
$$

Hence, the result follows from the fact that $f \in L^{2}(\mathbb{H})$.

Example 2.1. Consider $\mathcal{X}=\Pi$ and $J=\mathbb{Z}$. For any $\sigma \in \Pi$ and $j \in \mathbb{Z}$, define $\Lambda_{\sigma, j}$ : $L^{2}(\mathbb{H}) \rightarrow L^{2}(\mathbb{R}) \otimes L^{2}(\mathbb{R})$ as $\Lambda_{\sigma, j}(f)=T_{\sigma} f(j)$. Then for every $f \in L^{2}(\mathbb{H})$, using Lemma 2.3 we get

$$
\begin{aligned}
\int_{\sigma \in \Pi} \sum_{j \in \mathbb{Z}}\left\|\Lambda_{\sigma, j}(f)\right\|_{H . S .}^{2} d \sigma & =\int_{\sigma \in \Pi} \sum_{j \in \mathbb{Z}}\left\|T_{\sigma} f(j)\right\|_{H . S .}^{2} d \sigma \\
& =\int_{\sigma \in \Pi} \sum_{j \in \mathbb{Z}}\left\||\sigma+j|^{\frac{1}{2}} \widehat{f}(\sigma+j)\right\|_{H . S .}^{2} d \sigma \\
& =\|f\|_{L^{2}(\mathbb{H})}^{2} .
\end{aligned}
$$

Therefore $\left\{\Lambda_{\sigma, j}: \sigma \in \Pi, j \in \mathbb{Z}\right\}$ is a semi-continuous $g$-frame with frame bounds $A=$ $B=1$.

Corollary 2.4. Let $0<\mu(\mathcal{X})<\infty$. For any fixed $\sigma \in \mathcal{X}$, let $\left\{\Lambda_{\sigma, j}: j \in J\right\}$ be a g-frame for $L^{2}(\mathbb{H})$. Then $\left\{\Lambda_{\sigma, j}: \sigma \in \mathcal{X}, j \in J\right\}$ is a semi-continuous g-frame for $L^{2}(\mathbb{H})$ with respect to $(\mathcal{X}, \mu)$ with unified frame bounds multiplied by $\mu(\mathcal{X})$.

Proof. Since $\left\{\Lambda_{\sigma, j}: j \in J\right\}$ be a $g$-frame for $L^{2}(\mathbb{H})$, there exist constants $A, B>0$ such that

$$
A\|f\|_{L^{2}(\mathbb{H})}^{2} \leq \sum_{j \in J}\left\|\Lambda_{\sigma, j}(f)\right\|^{2} \leq B\|f\|_{L^{2}(\mathbb{H})}^{2}, \quad \forall f \in L^{2}(\mathbb{H}) .
$$

Taking integral from all sides of the preceding inequality, we obtain

$$
A \mu(\mathcal{X})\|f\|_{L^{2}(\mathbb{H})}^{2} \leq \int_{\mathcal{X}} \sum_{j \in J}\left\|\Lambda_{\sigma, j}(f)\right\|^{2} d \mu(\sigma) \leq B \mu(\mathcal{X})\|f\|_{L^{2}(\mathbb{H})}^{2}, \quad \forall f \in L^{2}(\mathbb{H}) .
$$

Hence, the result follows.

Now, we shall define shift-invariant spaces and give an example.

Definition 2.5. Let $\Gamma$ be a countable subset of $\mathbb{H}$. A subspace $\mathcal{V} \subset L^{2}(\mathbb{H})$ is called $\Gamma$-invariant if $L_{\gamma} \phi \in \mathcal{V}$ for all $\gamma \in \Gamma$ and all $\phi \in \mathcal{V}$, where $L_{\gamma} \phi(w)=\phi\left(\gamma^{-1} w\right), w \in \mathbb{H}$. If $\Gamma$ is a discrete subset of $\mathbb{H}$, then $\mathcal{V}$ is called shift-invariant.

Example 2.2. Let $\phi \in L^{2}(\mathbb{H})$ and $\Gamma$ be a lattice. Then the space $\langle\phi\rangle_{\Gamma}$ generated by $\Gamma$-shifts of $\phi$ is a shift-invariant space. 
Before we prove the main result of this section, we first need the following.

Let $T: L^{2}(\mathbb{H}) \rightarrow L^{2}(\Pi, \mathfrak{L})$. Then for any $\sigma \in \Pi$ and $j \in \mathbb{Z}, T f(\sigma)(j) \in L^{2}(\mathbb{R}) \otimes L^{2}(\mathbb{R})$. By Lemma 2.3, it is clear that $T f(\sigma)=T_{\sigma} f$. Let

$$
\Gamma=\Gamma_{1} \Gamma_{0}=\left\{x z \in \mathbb{H}: x \in \Gamma_{1}, z \in \Gamma_{0}\right\},
$$

where $\Gamma_{1}$ be any discrete subset of $\mathbb{H}$ and $\Gamma_{0}$ be the lattice of integral points in $\mathbb{Z}$. Then for $y \in \mathbb{H}$ and $\sigma \in \Pi$, define the unitary operator $\tilde{\rho}_{\sigma}(y): \mathfrak{L} \rightarrow \mathfrak{L}$ by

$$
\left(\tilde{\rho}_{\sigma}(y) h\right)_{j}=\rho_{\sigma+j}(y) \circ h_{j}, \quad h \in \mathfrak{L},
$$

where $\rho_{\sigma+j}(y) \circ h_{j}$ denotes function composition. Also, define $\tilde{\rho}(y): L^{2}(\Pi, \mathfrak{L}) \rightarrow L^{2}(\Pi, \mathfrak{L})$ by

$$
(\tilde{\rho}(y) a)(\sigma)=\tilde{\rho}_{\sigma}(y) a(\sigma), \quad a \in L^{2}(\Pi, \mathfrak{L}) .
$$

Note that if $\gamma \in \Gamma_{0}$, then $(\tilde{\rho}(\gamma) a)(\sigma)=e^{2 \pi i\langle\sigma, \gamma\rangle} a(\sigma)$ for all $a \in L^{2}(\Pi, \mathfrak{L})$. Further, the mapping $T$ is unitary, and for each $y \in \mathbb{H}$, we have

$$
T\left(L_{y} \phi\right)(\sigma)=(\tilde{\rho}(y) T \phi)(\sigma) .
$$

Proofs of these results and a more detailed study of these operators can be found in ([10], Section 3). Fix a discrete subset $\Gamma$ of $\mathbb{H}$ of the form $\Gamma_{1} \Gamma_{0}$. Let $\mathcal{V} \subset L^{2}(\mathbb{H})$ be a countable set. Define $E(\mathcal{V})=\left\{L_{\gamma} \phi: \gamma \in \Gamma, \phi \in \mathcal{V}\right\}$ and put $\mathcal{S}=\overline{\operatorname{span}} E(\mathcal{V})$. Let $R$ be the range function associated with $\mathcal{S}$. Motivated by the results of Currey et al. [10], we obtain the following.

Lemma 2.6. Let $f \in L^{2}(\mathbb{H}), \Gamma \subseteq \mathbb{H}$ and $\mathcal{V} \subset L^{2}(\mathbb{H})$. Then

$$
\sum_{\phi \in \mathcal{V}, \gamma \in \Gamma}\left|\left\langle f, L_{\gamma} \phi\right\rangle\right|^{2}=\int_{\Pi} \sum_{\phi \in \mathcal{V}, k \in \Gamma_{1}}\left|\left\langle T f(\sigma), T\left(L_{k} \phi\right)(\sigma)\right\rangle\right|^{2} d \sigma
$$

Proof. Let $f \in L^{2}(\mathbb{H})$. Since $\|T f\|=\|f\|$, we have

$$
\begin{aligned}
\sum_{\phi \in \mathcal{V}, \gamma \in \Gamma}\left|\left\langle f, L_{\gamma} \phi\right\rangle\right|^{2}=\sum_{\phi \in \mathcal{V}, \gamma \in \Gamma}\left|\left\langle T f, T\left(L_{\gamma} \phi\right)\right\rangle\right|^{2} & =\sum_{\phi \in \mathcal{V}, \gamma \in \Gamma}\left|\int_{\Pi}\left\langle T f(\sigma), T\left(L_{\gamma} \phi\right)(\sigma)\right\rangle d \sigma\right|^{2} \\
& =\sum_{\phi \in \mathcal{V}, \gamma \in \Gamma}\left|\int_{\Pi}\langle T f(\sigma),(\tilde{\rho}(\gamma) T \phi)(\sigma)\rangle d \sigma\right|^{2} .
\end{aligned}
$$

Putting $\gamma=k l$, with $k \in \Gamma_{1}, l \in \Gamma_{0}$, we get

$$
(\tilde{\rho}(k l) T \phi)(\sigma)=\tilde{\rho}_{\sigma}(k l) T \phi(\sigma)=\tilde{\rho}_{\sigma}(k) \tilde{\rho}_{\sigma}(l) T \phi(\sigma)=e^{2 \pi i\langle\sigma, l\rangle} \tilde{\rho}_{\sigma}(k) T \phi(\sigma) .
$$

Thus

$$
\sum_{\phi \in \mathcal{V}, \gamma \in \Gamma}\left|\int_{\Pi}\langle T f(\sigma),(\tilde{\rho}(\gamma) T \phi)(\sigma)\rangle d \sigma\right|^{2}=\sum_{\phi \in \mathcal{V},(k, l) \in \Gamma}\left|\int_{\Pi}\left\langle T f(\sigma), \tilde{\rho}_{\sigma}(k) T \phi(\sigma)\right\rangle e^{-2 \pi i\langle\sigma, l\rangle} d \sigma\right|^{2} .
$$

For each $k$, define $F_{k}(\sigma)=\left\langle T f(\sigma), \tilde{\rho}_{\sigma}(k) T \phi(\sigma)\right\rangle$. Then $F_{k}$ is integrable with square summable Fourier coefficients, hence $F_{k} \in L^{2}(\Pi)$. Using Fourier inversion formula we obtain

$$
\begin{aligned}
\sum_{\phi \in \mathcal{V},(k, l) \in \Gamma}\left|\int_{\Pi}\left\langle T f(\sigma), \tilde{\rho}_{\sigma}(k) T \phi(\sigma)\right\rangle e^{-2 \pi i\langle\sigma, l\rangle} d \sigma\right|^{2} & =\sum_{\phi \in \mathcal{V},(k, l) \in \Gamma}\left|\hat{F}_{k}(l)\right|^{2} \\
& =\sum_{\phi \in \mathcal{V}, k \in \Gamma_{1}}\left\|F_{k}\right\|^{2} \\
& =\sum_{\phi \in \mathcal{V}, k \in \Gamma_{1}} \int_{\Pi}\left|F_{k}(\sigma)\right|^{2} d \sigma .
\end{aligned}
$$


Again, by substituting $F_{k}(\sigma)=\left\langle T f(\sigma), \tilde{\rho}_{\sigma}(k) T \phi(\sigma)\right\rangle$ in the above we get

$$
\begin{aligned}
\sum_{\phi \in \mathcal{V}, \gamma \in \Gamma}\left|\left\langle f, L_{\gamma} \phi\right\rangle\right|^{2} & =\sum_{\phi \in \mathcal{V}, k \in \Gamma_{1}} \int_{\Pi}\left|F_{k}(\sigma)\right|^{2} d \sigma=\sum_{\phi \in \mathcal{V}, k \in \Gamma_{1}} \int_{\Pi}\left|\left\langle T f(\sigma), \tilde{\rho}_{\sigma}(k) T \phi(\sigma)\right\rangle\right|^{2} d \sigma \\
& =\int_{\Pi} \sum_{\phi \in \mathcal{V}, k \in \Gamma_{1}}\left|\left\langle T f(\sigma), T\left(L_{k} \phi\right)(\sigma)\right\rangle\right|^{2} d \sigma
\end{aligned}
$$

This completes the proof.

Now we are in a position to prove our main result of this section.

Theorem 2.7. If $\left\{T_{\sigma}\left(L_{k} \phi\right): k \in \Gamma_{1}, \phi \in \mathcal{V}\right\}$ is a frame for its spanned vector space for almost every $\sigma \in \Pi$. Then $\left\{L_{\gamma} \phi: \gamma \in \Gamma, \phi \in \mathcal{V}\right\}$ is also a frame for its spanned vector space.

Proof. Suppose that $f \in \mathcal{S}$, then $T f(\sigma) \in R(\sigma)$ holds for a.e. $\sigma$. Since for a.e. $\sigma \in \Pi$, $\left\{T_{\sigma}\left(L_{k} \phi\right): k \in \Gamma_{1}, \phi \in \mathcal{V}\right\}$ is a frame for its spanned vector space, there exist $0<A \leq$ $B<\infty$ such that

$$
A\|T f(\sigma)\|^{2} \leq \int_{\Pi} \sum_{\phi \in \mathcal{V}, k \in \Gamma_{1}}\left|\left\langle T f(\sigma), T\left(L_{k} \phi\right)(\sigma)\right\rangle\right|^{2} d \sigma \leq B\|T f(\sigma)\|^{2} .
$$

holds for a.e. $\sigma$. Integrating over $\Pi$ yields

$$
\begin{aligned}
A\|f\|^{2}=A\|T f\|^{2}=A \int_{\Pi}\|T f(\sigma)\|^{2} d \sigma & \leq \int_{\Pi} \sum_{\phi \in \mathcal{V}, k \in \Gamma_{1}}\left|\left\langle T f(\sigma), T\left(L_{k} \phi\right)(\sigma)\right\rangle\right|^{2} d \sigma \\
& \leq B \int_{\Pi}\|T f(\sigma)\|^{2} d \sigma=B\|f\|^{2} .
\end{aligned}
$$

Using (2.6) we obtain

$$
A\|f\|^{2} \leq \sum_{\phi \in \mathcal{V}, \gamma \in \Gamma}\left|\left\langle f, L_{\gamma} \phi\right\rangle\right|^{2} \leq B\|f\|^{2}
$$

Hence, we have the desired result.

Remark 2.8. Notice that the family $\left\{T_{\sigma}\left(L_{k} \phi\right): k \in \Gamma_{1}, \phi \in \mathcal{V}\right\}$ constitutes a frame for the space which consists of all functions of the form $T_{\sigma} f$ for every $f \in L^{2}(\mathbb{H})$. Similarly, the above result can be extended for semi-continuous $g$-frames using the Riesz representation theorem.

\section{IDENTities AND INEQUALities FOR SEMI-CONTINUOUS G-FRAMES}

Let $\left\{\Lambda_{x, j} \in \mathcal{L}\left(\mathcal{H}, \mathcal{K}_{x, j}\right): x \in \mathcal{X}, j \in J\right\}$ be a semi-continuous $g$-frame for $\mathcal{H}$ with respect to $(\mathcal{X}, \mu)$. Then we define the semi-continuous $g$-frame operator $S$ as follows:

$$
S: \mathcal{H} \rightarrow \mathcal{H}, \quad S f=\int_{\mathcal{X}} \sum_{j \in J} \Lambda_{x, j}^{*} \Lambda_{x, j} f d \mu(x),
$$

where $\Lambda_{x, j}^{*}$ is the adjoint of $\Lambda_{x, j}$. It is easy to show that $S$ is a bounded, invertible, self-adjoint and positive operator. Therefore for any $f \in \mathcal{H}$, we have the following reconstructions:

$$
\begin{aligned}
& f=S S^{-1} f=\int_{\mathcal{X}} \sum_{j \in J} \Lambda_{x, j}^{*} \Lambda_{x, j} S^{-1} f d \mu(x), \\
& f=S^{-1} S f=\int_{\mathcal{X}} \sum_{j \in J} S^{-1} \Lambda_{x, j}^{*} \Lambda_{x, j} f d \mu(x) .
\end{aligned}
$$

Denote $\tilde{\Lambda}_{x, j}=\Lambda_{x, j} S^{-1}$. Then $\left\{\tilde{\Lambda}_{x, j}: x \in \mathcal{X}, j \in J\right\}$ is also a semi-continuous $g$-frame with frame bounds $\frac{1}{B}, \frac{1}{A}$, which we call the canonical dual frame of $\left\{\Lambda_{x, j}: x \in \mathcal{X}, j \in J\right\}$. 
A semi-continuous $g$-frame $\left\{\mathcal{G}_{x, j} \in \mathcal{L}\left(\mathcal{H}, \mathcal{K}_{x, j}\right): x \in \mathcal{X}, j \in J\right\}$ is called an alternate dual frame of $\left\{\Lambda_{x, j}: x \in \mathcal{X}, j \in J\right\}$ if for all $f \in \mathcal{H}$, the following identity holds:

$$
f=\int_{\mathcal{X}} \sum_{j \in J} \Lambda_{x, j}^{*} \mathcal{G}_{x, j} f d \mu(x)=\int_{\mathcal{X}} \sum_{j \in J} \mathcal{G}_{x, j}^{*} \Lambda_{x, j} f d \mu(x) .
$$

A semi-continuous $g$-frame $\left\{\Lambda_{x, j}: x \in \mathcal{X}, j \in J\right\}$ is called a Parseval semi-continuous $g$-frame, if the frame bounds $A=B=1$. For any $\mathcal{X}_{1} \subset \mathcal{X}$, we denote $\mathcal{X}_{1}^{c}=\mathcal{X} \backslash \mathcal{X}_{1}$, and define the following operator:

$$
S_{\mathcal{X}_{1}} f=\int_{\mathcal{X}_{1}} \sum_{j \in J} \Lambda_{x, j}^{*} \Lambda_{x, j} f d \mu(x)
$$

In [4], the authors proved a longstanding conjecture of the signal processing community: a signal can be reconstructed without information about the phase. While working on efficient algorithms for signal reconstruction, Balan et al. [5] discovered a remarkable new identity for Parseval discrete frames, given in the following form.

Theorem 3.1. Let $\left\{f_{j}\right\}_{j \in J}$ be a Parseval frame for $\mathcal{H}$, then for every $K \subset J$ and every $f \in \mathcal{H}$, we have

$$
\sum_{j \in K}\left|\left\langle f, f_{j}\right\rangle\right|^{2}-\left\|\sum_{j \in K}\left\langle f, f_{j}\right\rangle f_{j}\right\|^{2}=\sum_{j \in K^{c}}\left|\left\langle f, f_{j}\right\rangle\right|^{2}-\left\|\sum_{j \in K^{c}}\left\langle f, f_{j}\right\rangle f_{j}\right\|^{2} .
$$

Theorem 3.2. If $\left\{f_{j}\right\}_{j \in J}$ be a Parseval frame for $\mathcal{H}$, then for every $K \subset J$ and every $f \in \mathcal{H}$, we have

$$
\sum_{j \in K}\left|\left\langle f, f_{j}\right\rangle\right|^{2}+\left\|\sum_{j \in K^{c}}\left\langle f, f_{j}\right\rangle f_{j}\right\|^{2} \geq \frac{3}{4}\|f\|^{2} .
$$

In fact, the identity appears in Theorem 3.1 was obtained in [5] as a particular case of the following result for general frames.

Theorem 3.3. Let $\left\{f_{j}\right\}_{j \in J}$ be a frame for $\mathcal{H}$ with canonical dual frame $\left\{\tilde{f}_{j}\right\}_{j \in J}$. Then for every $K \subset J$ and every $f \in \mathcal{H}$, we have

$$
\sum_{j \in K}\left|\left\langle f, f_{j}\right\rangle\right|^{2}-\sum_{j \in J}\left|\left\langle S_{K} f, \tilde{f}_{j}\right\rangle\right|^{2}=\sum_{j \in K^{c}}\left|\left\langle f, f_{j}\right\rangle\right|^{2}-\sum_{j \in J}\left|\left\langle S_{K^{c}} f, \tilde{f}_{j}\right\rangle\right|^{2}
$$

Motivated by these interesting results, the authors in $[18,30]$ generalized Theorems 3.1 and 3.2 to canonical and alternate dual frames. In this section, we investigate the above mentioned results for semi-continuous $g$-frames and derive some important identities and inequalities of these frames. We first state a simple result on operators.

Lemma 3.4. [30] If $P, Q \in \mathcal{L}(\mathcal{H})$ satisfying $P+Q=I$, then $P-P^{*} P=Q^{*}-Q^{*} Q$.

Proof. We compute $P-P^{*} P=\left(I-P^{*}\right) P=Q^{*}(I-Q)=Q^{*}-Q^{*} Q$.

Theorem 3.5. Let $\left\{\Lambda_{x, j}: x \in \mathcal{X}, j \in J\right\}$ be a Parseval semi-continuous g-frame for $\mathcal{H}$ with respect to $(\mathcal{X}, \mu)$. Then for every $\mathcal{X}_{1} \subset \mathcal{X}$ and every $f \in \mathcal{H}$, we have

$$
\begin{aligned}
\int_{\mathcal{X}_{1}} \sum_{j \in J}\left\|\Lambda_{x, j} f\right\|^{2} d \mu(x) & -\left\|\int_{\mathcal{X}_{1}} \sum_{j \in J} \Lambda_{x, j}^{*} \Lambda_{x, j} f d \mu(x)\right\|^{2} \\
& =\int_{\mathcal{X}_{1}^{c}} \sum_{j \in J}\left\|\Lambda_{x, j} f\right\|^{2} d \mu(x)-\left\|\int_{\mathcal{X}_{1}^{c}} \sum_{j \in J} \Lambda_{x, j}^{*} \Lambda_{x, j} f d \mu(x)\right\|^{2} .
\end{aligned}
$$


Proof. Since $\left\{\Lambda_{x, j}: x \in \mathcal{X}, j \in J\right\}$ is a Parseval semi-continuous $g$-frame, the corresponding frame operator $S=I$, and hence $S_{\mathcal{X}_{1}}+S_{\mathcal{X}_{1}^{c}}=I$. Note that $S_{\mathcal{X}_{1}^{c}}$ is a self-adjoint operator, and therefore $S_{\mathcal{X}_{1}^{c}}^{*}=S_{\mathcal{X}_{1}^{c}}$. Applying Lemma 3.4 to the operators $S_{\mathcal{X}_{1}}$ and $S_{\mathcal{X}_{1}^{c}}$, we obtain that for every $f \in \mathcal{H}$

$$
\begin{aligned}
\left\langle S_{\mathcal{X}_{1}} f, f\right\rangle-\left\langle S_{\mathcal{X}_{1}}^{*} S_{\mathcal{X}_{1}} f, f\right\rangle=\left\langle S_{\mathcal{X}_{1}^{c}}^{*} f, f\right\rangle-\left\langle S_{\mathcal{X}_{1}^{c}}^{*} S_{\mathcal{X}_{1}^{c}} f, f\right\rangle & \\
& \Rightarrow\left\langle S_{\mathcal{X}_{1}} f, f\right\rangle-\left\|S_{\mathcal{X}_{1}} f\right\|^{2}=\left\langle S_{\mathcal{X}_{1}^{c}} f, f\right\rangle-\left\|S_{\mathcal{X}_{1}^{c}} f\right\|^{2} .
\end{aligned}
$$

We have

$$
\begin{aligned}
\left\langle S_{\mathcal{X}_{1}} f, f\right\rangle=\left\langle\int_{\mathcal{X}_{1}} \sum_{j \in J} \Lambda_{x, j}^{*} \Lambda_{x, j} f d \mu(x), f\right\rangle & =\int_{\mathcal{X}_{1}} \sum_{j \in J}\left\langle\Lambda_{x, j} f, \Lambda_{x, j} f\right\rangle d \mu(x) \\
& =\int_{\mathcal{X}_{1}} \sum_{j \in J}\left\|\Lambda_{x, j} f\right\|^{2} d \mu(x) .
\end{aligned}
$$

Similarly

$$
\left\langle S_{\mathcal{X}_{1}^{c}} f, f\right\rangle=\int_{\mathcal{X}_{1}^{c}} \sum_{j \in J}\left\|\Lambda_{x, j} f\right\|^{2} d \mu(x) .
$$

Using equations (3.10) and (3.11) in (3.9), we obtain the desired result.

Now we generalize Theorem 3.1 to dual semi-continuous $g$-frames. We first need the following lemma.

Lemma 3.6. [25] Let $P, Q \in \mathcal{L}(\mathcal{H})$ be two self-adjoint operators such that $P+Q=I$. Then for any $\lambda \in[0,1]$ and every $f \in \mathcal{H}$ we have

$$
\|P f\|^{2}+2 \lambda\langle Q f, f\rangle=\|Q f\|^{2}+2(1-\lambda)\langle P f, f\rangle+(2 \lambda-1)\|f\|^{2} \geq\left(1-(\lambda-1)^{2}\right)\|f\|^{2} .
$$

Theorem 3.7. Let $\left\{\Lambda_{x, j}: x \in \mathcal{X}, j \in J\right\}$ be a semi-continuous $g$-frame for $\mathcal{H}$ with respect to $(\mathcal{X}, \mu)$ and $\left\{\tilde{\Lambda}_{x, j}: x \in \mathcal{X}, j \in J\right\}$ be the canonical dual frame of $\left\{\Lambda_{x, j}: x \in \mathcal{X}, j \in J\right\}$. Then for any $\lambda \in[0,1]$, for every $\mathcal{X}_{1} \subset \mathcal{X}$ and every $f \in \mathcal{H}$, we have

$$
\begin{aligned}
\int_{\mathcal{X}} \sum_{j \in J}\left\|\tilde{\Lambda}_{x, j} S_{\mathcal{X}_{1}} f\right\|^{2} d \mu(x)+\int_{\mathcal{X}_{1}^{c}} \sum_{j \in J}\left\|\Lambda_{x, j} f\right\|^{2} d \mu(x) \\
\quad=\int_{\mathcal{X}} \sum_{j \in J}\left\|\tilde{\Lambda}_{x, j} S_{\mathcal{X}_{1}^{c}} f\right\|^{2} d \mu(x)+\int_{\mathcal{X}_{1}} \sum_{j \in J}\left\|\Lambda_{x, j} f\right\|^{2} d \mu(x) \\
\geq\left(2 \lambda-\lambda^{2}\right) \int_{\mathcal{X}_{1}} \sum_{j \in J}\left\|\Lambda_{x, j} f\right\|^{2} d \mu(x)+\left(1-\lambda^{2}\right) \int_{\mathcal{X}_{1}^{c}} \sum_{j \in J}\left\|\Lambda_{x, j} f\right\|^{2} d \mu(x) .
\end{aligned}
$$

Proof. Let $S$ be the frame operator for $\left\{\Lambda_{x, j}: x \in \mathcal{X}, j \in J\right\}$. Since $S_{\mathcal{X}_{1}}+S_{\mathcal{X}_{1}^{c}}=S$, it follows that $S^{-1 / 2} S_{\mathcal{X}_{1}} S^{-1 / 2}+S^{-1 / 2} S_{\mathcal{X}_{1}^{c}} S^{-1 / 2}=I$. Considering $P=S^{-1 / 2} S_{\mathcal{X}_{1}} S^{-1 / 2}$, $Q=S^{-1 / 2} S_{\mathcal{X}_{1}^{c}} S^{-1 / 2}$, and $S^{1 / 2} f$ instead of $f$ in Lemma 3.6, we obtain

$$
\begin{array}{r}
\left\|S^{-1 / 2} S_{\mathcal{X}_{1}} f\right\|^{2}+2 \lambda\left\langle S^{-1 / 2} S_{\mathcal{X}_{1}^{c}} f, S^{1 / 2} f\right\rangle \\
=\left\|S^{-1 / 2} S_{\mathcal{X}_{1}^{c}} f\right\|^{2}+2(1-\lambda)\left\langle S^{-1 / 2} S_{\mathcal{X}_{1}} f, S^{1 / 2} f\right\rangle+(2 \lambda-1)\left\|S^{1 / 2} f\right\|^{2} \\
\quad \geq\left(1-(\lambda-1)^{2}\right)\left\|S^{1 / 2} f\right\|^{2} \\
\Rightarrow\left\langle S^{-1} S_{\mathcal{X}_{1}} f, S_{\mathcal{X}_{1}} f\right\rangle+\left\langle S_{\mathcal{X}_{1}^{c}} f, f\right\rangle=\left\langle S^{-1} S_{\mathcal{X}_{1}^{c}} f, S_{\mathcal{X}_{1}^{c}} f\right\rangle+\left\langle S_{\mathcal{X}_{1}} f, f\right\rangle \\
\quad \geq\left(2 \lambda-\lambda^{2}\right)\left\langle S_{\mathcal{X}_{1}} f, f\right\rangle+\left(1-\lambda^{2}\right)\left\langle S_{\mathcal{X}_{1}^{c}} f, f\right\rangle .
\end{array}
$$


We have

$$
\begin{aligned}
\left\langle S^{-1} S_{\mathcal{X}_{1}} f, S_{\mathcal{X}_{1}} f\right\rangle & =\left\langle S S^{-1} S_{\mathcal{X}_{1}} f, S^{-1} S_{\mathcal{X}_{1}} f\right\rangle \\
& =\left\langle\int_{\mathcal{X}} \sum_{j \in J} \Lambda_{x, j}^{*} \Lambda_{x, j} S^{-1} S_{\mathcal{X}_{1}} f d \mu(x), S^{-1} S_{\mathcal{X}_{1}} f\right\rangle \\
& =\int_{\mathcal{X}} \sum_{j \in J}\left\langle\Lambda_{x, j} S^{-1} S_{\mathcal{X}_{1}} f, \Lambda_{x, j} S^{-1} S \mathcal{X}_{1} f\right\rangle d \mu(x) \\
& =\int_{\mathcal{X}} \sum_{j \in J}\left\langle\tilde{\Lambda}_{x, j} S_{\mathcal{X}_{1}} f, \tilde{\Lambda}_{x, j} S_{\mathcal{X}_{1}} f\right\rangle d \mu(x) \\
& =\int_{\mathcal{X}} \sum_{j \in J}\left\|\tilde{\Lambda}_{x, j} S_{\mathcal{X}_{1}} f\right\|^{2} d \mu(x) .
\end{aligned}
$$

Similarly

$$
\begin{gathered}
\left\langle S^{-1} S_{\mathcal{X}_{1}^{c}} f, S_{\mathcal{X}_{1}^{c}} f\right\rangle=\int_{\mathcal{X}} \sum_{j \in J}\left\|\tilde{\Lambda}_{x, j} S_{\mathcal{X}_{1}^{c}} f\right\|^{2} d \mu(x) . \\
\left\langle S_{\mathcal{X}_{1}^{c}} f, f\right\rangle=\int_{\mathcal{X}_{1}^{c}} \sum_{j \in J}\left\|\Lambda_{x, j} f\right\|^{2} d \mu(x) . \\
\left\langle S_{\mathcal{X}_{1}} f, f\right\rangle=\int_{\mathcal{X}_{1}} \sum_{j \in J}\left\|\Lambda_{x, j} f\right\|^{2} d \mu(x) .
\end{gathered}
$$

Using equations (3.13)-(3.16) in the inequality (3.12), we obtain the desired result.

Lemma 3.8. [25] If $P, Q \in \mathcal{L}(\mathcal{H})$ satisfy $P+Q=I$, then for any $\lambda \in[0,1]$ and every $f \in \mathcal{H}$ we have

$$
P^{*} P+\lambda\left(Q^{*}+Q\right)=Q^{*} Q+(1-\lambda)\left(P^{*}+P\right)+(2 \lambda-1) I \geq\left(1-(\lambda-1)^{2}\right) I .
$$

Theorem 3.9. Let $\left\{\Lambda_{x, j}: x \in \mathcal{X}, j \in J\right\}$ be a semi-continuous $g$-frame for $\mathcal{H}$ with respect to $(\mathcal{X}, \mu)$ and $\left\{\mathcal{G}_{x, j}: x \in \mathcal{X}, j \in J\right\}$ be an alternate dual frame of $\left\{\Lambda_{x, j}: x \in \mathcal{X}, j \in J\right\}$. Then for any $\lambda \in[0,1]$, for every $\mathcal{X}_{1} \subset \mathcal{X}$ and every $f \in \mathcal{H}$, we have

$$
\begin{aligned}
& \operatorname{Re}\left\{\int_{\mathcal{X}_{1}^{c}} \sum_{j \in J}\left\langle\mathcal{G}_{x, j} f, \Lambda_{x, j} f\right\rangle d \mu(x)\right\}+\left\|\int_{\mathcal{X}_{1}} \sum_{j \in J} \Lambda_{x, j}^{*} \mathcal{G}_{x, j} f d \mu(x)\right\|^{2} \\
& =\operatorname{Re}\left\{\int_{\mathcal{X}_{1}} \sum_{j \in J}\left\langle\mathcal{G}_{x, j} f, \Lambda_{x, j} f\right\rangle d \mu(x)\right\}+\left\|\int_{\mathcal{X}_{1}^{c}} \sum_{j \in J} \Lambda_{x, j}^{*} \mathcal{G}_{x, j} f d \mu(x)\right\|^{2} \\
& \geq\left(2 \lambda-\lambda^{2}\right) \operatorname{Re}\left\{\int_{\mathcal{X}_{1}} \sum_{j \in J}\left\langle\mathcal{G}_{x, j} f, \Lambda_{x, j} f\right\rangle d \mu(x)\right\} \\
& \quad+\left(1-\lambda^{2}\right) \operatorname{Re}\left\{\int_{\mathcal{X}_{1}^{c}} \sum_{j \in J}\left\langle\mathcal{G}_{x, j} f, \Lambda_{x, j} f\right\rangle d \mu(x)\right\} .
\end{aligned}
$$

Proof. For $\mathcal{X}_{1} \subset \mathcal{X}$ and $f \in \mathcal{H}$, define the operator $F_{\mathcal{X}_{1}}$ by

$$
F_{\mathcal{X}_{1}} f=\int_{\mathcal{X}_{1}} \sum_{j \in J} \Lambda_{x, j}^{*} \mathcal{G}_{x, j} f d \mu(x) .
$$


Then $F_{\mathcal{X}_{1}} \in \mathcal{L}(\mathcal{H})$. By $(3.7)$, we have $F_{\mathcal{X}_{1}}+F_{\mathcal{X}_{1}^{c}}=I$. By Lemma 3.8 , we get

$$
\begin{aligned}
& \left(1-(\lambda-1)^{2}\right)\|f\|^{2} \leq\left\langle F_{\mathcal{X}_{1}}^{*} F_{\mathcal{X}_{1}} f, f\right\rangle+\lambda\left\langle\left(F_{\mathcal{X}_{1}^{c}}^{*}+F_{\mathcal{X}_{1}^{c}}\right) f, f\right\rangle \\
& =\left\langle F_{\mathcal{X}_{1}^{c}}^{*} F_{\mathcal{X}_{1}^{c}} f, f\right\rangle+(1-\lambda)\left\langle\left(F_{\mathcal{X}_{1}}^{*}+F_{\mathcal{X}_{1}}\right) f, f\right\rangle+(2 \lambda-1)\|f\|^{2} \\
& \Rightarrow\left(2 \lambda-\lambda^{2}\right) \operatorname{Re}(\langle I f, f\rangle) \leq\left\|F_{\mathcal{X}_{1}} f\right\|^{2}+\lambda\left(\overline{\left\langle F_{\mathcal{X}_{1}^{c}} f, f\right\rangle}+\left\langle F_{\mathcal{X}_{1}^{c}} f, f\right\rangle\right) \\
& =\left\|F_{\mathcal{X}_{1}^{c}} f\right\|^{2}+(1-\lambda)\left(\overline{\left\langle F_{\mathcal{X}_{1}} f, f\right\rangle}+\left\langle F_{\mathcal{X}_{1}} f, f\right\rangle\right)+(2 \lambda-1)\|f\|^{2} \\
& \Rightarrow\left(2 \lambda-\lambda^{2}\right) \operatorname{Re}\left(\left\langle F_{\mathcal{X}_{1}} f, f\right\rangle\right)+\left(1-\lambda^{2}\right) \operatorname{Re}\left(\left\langle F_{\mathcal{X}_{1}^{c}} f, f\right\rangle\right) \leq\left\|F_{\mathcal{X}_{1}} f\right\|^{2}+\operatorname{Re}\left(\left\langle F_{\mathcal{X}_{1}^{c}} f, f\right\rangle\right) \\
& =\left\|F_{\mathcal{X}_{1}^{c}} f\right\|^{2}+\operatorname{Re}\left(\left\langle F_{\mathcal{X}_{1}} f, f\right\rangle\right) \text {. }
\end{aligned}
$$

We have

$$
\begin{gathered}
\left\langle F_{\mathcal{X}_{1}} f, f\right\rangle=\left\langle\int_{\mathcal{X}_{1}} \sum_{j \in J} \Lambda_{x, j}^{*} \mathcal{G}_{x, j} f d \mu(x), f\right\rangle=\int_{\mathcal{X}_{1}} \sum_{j \in J}\left\langle\mathcal{G}_{x, j} f, \Lambda_{x, j} f\right\rangle d \mu(x) . \\
\left\langle F_{\mathcal{X}_{1}^{c}} f, f\right\rangle=\int_{\mathcal{X}_{1}^{c}} \sum_{j \in J}\left\langle\mathcal{G}_{x, j} f, \Lambda_{x, j} f\right\rangle d \mu(x) .
\end{gathered}
$$

Using equations (3.19), (3.20) and (3.17) in (3.18), we obtain the desired inequality.

Next we give a generalization of the above theorem to a more general form that does not involve the real parts of the complex numbers.

Theorem 3.10. Let $\left\{\Lambda_{x, j}: x \in \mathcal{X}, j \in J\right\}$ be a semi-continuous g-frame for $\mathcal{H}$ with respect to $(\mathcal{X}, \mu)$ and $\left\{\mathcal{G}_{x, j}: x \in \mathcal{X}, j \in J\right\}$ be an alternate dual frame of $\left\{\Lambda_{x, j}: x \in\right.$ $\mathcal{X}, j \in J\}$. Then for every $\mathcal{X}_{1} \subset \mathcal{X}$ and every $f \in \mathcal{H}$, we have

$$
\begin{aligned}
& \left(\int_{\mathcal{X}_{1}^{c}} \sum_{j \in J}\left\langle\mathcal{G}_{x, j} f, \Lambda_{x, j} f\right\rangle d \mu(x)\right)+\left\|\int_{\mathcal{X}_{1}} \sum_{j \in J} \Lambda_{x, j}^{*} \mathcal{G}_{x, j} f d \mu(x)\right\|^{2} \\
& =\overline{\left(\int_{\mathcal{X}_{1}} \sum_{j \in J}\left\langle\mathcal{G}_{x, j} f, \Lambda_{x, j} f\right\rangle d \mu(x)\right)}+\left\|\int_{\mathcal{X}_{1}^{c}} \sum_{j \in J} \Lambda_{x, j}^{*} \mathcal{G}_{x, j} f d \mu(x)\right\|^{2} .
\end{aligned}
$$

Proof. For $\mathcal{X}_{1} \subset \mathcal{X}$ and $f \in \mathcal{H}$, we define the operator $F_{\mathcal{X}_{1}}$ as in Theorem 3.9. Therefore, we have $F_{\mathcal{X}_{1}}+F_{\mathcal{X}_{1}^{c}}=I$. By Lemma 3.4, we have

$$
\begin{aligned}
& \left(\int_{\mathcal{X}_{1}^{c}} \sum_{j \in J}\left\langle\mathcal{G}_{x, j} f, \Lambda_{x, j} f\right\rangle d \mu(x)\right)+\left\|\int_{\mathcal{X}_{1}} \sum_{j \in J} \Lambda_{x, j}^{*} \mathcal{G}_{x, j} f d \mu(x)\right\|^{2} \\
& =\left\langle F_{\mathcal{X}_{1}^{c}} f, f\right\rangle+\left\langle F_{\mathcal{X}_{1}}^{*} F_{\mathcal{X}_{1}} f, f\right\rangle=\left\langle F_{\mathcal{X}_{1}}^{*} f, f\right\rangle+\left\langle F_{\mathcal{X}_{1}^{c}}^{*} F_{\mathcal{X}_{1}^{c}} f, f\right\rangle \\
& =\overline{\left\langle F_{\mathcal{X}_{1}} f, f\right\rangle}+\left\|F_{\mathcal{X}_{1}^{c}} f\right\|^{2} \\
& =\overline{\left(\int_{\mathcal{X}_{1}} \sum_{j \in J}\left\langle\mathcal{G}_{x, j} f, \Lambda_{x, j} f\right\rangle d \mu(x)\right)}+\left\|\int_{\mathcal{X}_{1}^{c}} \sum_{j \in J} \Lambda_{x, j}^{*} \mathcal{G}_{x, j} f d \mu(x)\right\|^{2} .
\end{aligned}
$$

Hence the relation stated in the theorem holds.

\section{Stability of Semi-Continuous g-Frames}

The stability of frames is important in practice, so it has received much attentions and is, therefore, studied widely by many authors (see $[8,26,28]$ ). In this section, we study the stability of semi-continuous $g$-frames. The following is a fundamental result in the study of the stability of frames. 
Proposition 4.1. ([6], Theorem 2) Let $\left\{f_{i}\right\}_{i=1}^{\infty}$ be a frame for some Hilbert space $\mathcal{H}$ with bounds $A, B$. Let $\left\{g_{i}\right\}_{i=1}^{\infty} \subseteq \mathcal{H}$ and assume that there exist constants $\lambda_{1}, \lambda_{2}, \mu \geq 0$ such that $\max \left(\lambda_{1}+\frac{\mu}{\sqrt{A}}, \lambda_{2}\right)<1$ and

$$
\left\|\sum_{i=1}^{n} c_{i}\left(f_{i}-g_{i}\right)\right\| \leq \lambda_{1}\left\|\sum_{i=1}^{n} c_{i} f_{i}\right\|+\lambda_{2}\left\|\sum_{i=1}^{n} c_{i} g_{i}\right\|+\mu\left[\sum_{i=1}^{n}\left|c_{i}\right|^{2}\right]^{1 / 2}
$$

for all $c_{1}, \ldots, c_{n}(n \in \mathbb{N})$. Then $\left\{g_{i}\right\}_{i=1}^{\infty}$ is a frame for $\mathcal{H}$ with bounds

$$
A\left(1-\frac{\lambda_{1}+\lambda_{2}+\frac{\mu}{\sqrt{A}}}{1+\lambda_{2}}\right)^{2}, B\left(1+\frac{\lambda_{1}+\lambda_{2}+\frac{\mu}{\sqrt{B}}}{1-\lambda_{2}}\right)^{2} .
$$

Similar to discrete frames, semi-continuous $g$-frames are stable under small perturbations. The stability of semi-continuous $g$-frames is discussed in the following theorem.

Theorem 4.2. Let $\left\{\Lambda_{x, j}: x \in \mathcal{X}, j \in J\right\}$ be a semi-continuous $g$-frame for $\mathcal{H}$ with respect to $(\mathcal{X}, \mu)$, with frame bounds $A$ and $B$. Suppose that $\Gamma_{x, j} \in \mathcal{L}\left(\mathcal{H}, \mathcal{K}_{x, j}\right)$ for any $x \in \mathcal{X}, j \in J$ and there exist constants $\lambda_{1}, \lambda_{2}, \mu \geq 0$ such that $\max \left(\lambda_{1}+\frac{\mu}{\sqrt{A}}, \lambda_{2}\right)<1$ and the following condition is satisfied

$$
\begin{aligned}
& \left(\int_{\mathcal{X}} \sum_{j \in J}\left\|\left(\Lambda_{x, j}-\Gamma_{x, j}\right) f\right\|^{2} d \mu(x)\right)^{1 / 2} \\
& \quad \leq \lambda_{1}\left(\int_{\mathcal{X}} \sum_{j \in J}\left\|\Lambda_{x, j}(f)\right\|^{2} d \mu(x)\right)^{1 / 2}+\lambda_{2}\left(\int_{\mathcal{X}} \sum_{j \in J}\left\|\Gamma_{x, j}(f)\right\|^{2} d \mu(x)\right)^{1 / 2}+\mu\|f\|,
\end{aligned}
$$

for all $f \in \mathcal{H}$. Then $\left\{\Gamma_{x, j}: x \in \mathcal{X}, j \in J\right\}$ is a semi-continuous $g$-frame for $\mathcal{H}$ with respect to $(\mathcal{X}, \mu)$, with frame bounds

$$
A\left(1-\frac{\lambda_{1}+\lambda_{2}+\frac{\mu}{\sqrt{A}}}{1+\lambda_{2}}\right)^{2}, B\left(1+\frac{\lambda_{1}+\lambda_{2}+\frac{\mu}{\sqrt{B}}}{1-\lambda_{2}}\right)^{2} .
$$

Proof. Notice that

$$
\int_{\mathcal{X}} \sum_{j \in J}\left\|\Lambda_{x, j}(f)\right\|^{2} d \mu(x) \leq B\|f\|^{2} .
$$

From (4.22) we see that

$$
\begin{aligned}
& \left(\int_{\mathcal{X}} \sum_{j \in J}\left\|\left(\Lambda_{x, j}-\Gamma_{x, j}\right) f\right\|^{2} d \mu(x)\right)^{1 / 2} \\
& \leq\left(\lambda_{1} \sqrt{B}+\mu\right)\|f\|+\lambda_{2}\left(\int_{\mathcal{X}} \sum_{j \in J}\left\|\Gamma_{x, j}(f)\right\|^{2} d \mu(x)\right)^{1 / 2} .
\end{aligned}
$$

Using the triangle inequality, we get

$$
\begin{aligned}
& \left(\int_{\mathcal{X}} \sum_{j \in J}\left\|\left(\Lambda_{x, j}-\Gamma_{x, j}\right) f\right\|^{2} d \mu(x)\right)^{1 / 2} \\
& \geq\left(\int_{\mathcal{X}} \sum_{j \in J}\left\|\Gamma_{x, j}(f)\right\|^{2} d \mu(x)\right)^{1 / 2}-\left(\int_{\mathcal{X}} \sum_{j \in J}\left\|\Lambda_{x, j}(f)\right\|^{2} d \mu(x)\right)^{1 / 2} .
\end{aligned}
$$


Hence

$$
\begin{aligned}
& \left(1-\lambda_{2}\right)\left(\int_{\mathcal{X}} \sum_{j \in J}\left\|\Gamma_{x, j}(f)\right\|^{2} d \mu(x)\right)^{1 / 2} \\
& \leq\left(\lambda_{1} \sqrt{B}+\mu\right)\|f\|+\left(\int_{\mathcal{X}} \sum_{j \in J}\left\|\Lambda_{x, j}(f)\right\|^{2} d \mu(x)\right)^{1 / 2} \leq \sqrt{B}\left(1+\lambda_{1}+\frac{\mu}{\sqrt{B}}\right)\|f\| .
\end{aligned}
$$

Therefore

$$
\int_{\mathcal{X}} \sum_{j \in J}\left\|\Gamma_{x, j}(f)\right\|^{2} d \mu(x) \leq B\left(1+\frac{\lambda_{1}+\lambda_{2}+\frac{\mu}{\sqrt{B}}}{1-\lambda_{2}}\right)^{2}\|f\|^{2} .
$$

Similarly, we can prove that

$$
\int_{\mathcal{X}} \sum_{j \in J}\left\|\Gamma_{x, j}(f)\right\|^{2} d \mu(x) \geq A\left(1-\frac{\lambda_{1}+\lambda_{2}+\frac{\mu}{\sqrt{A}}}{1+\lambda_{2}}\right)^{2}\|f\|^{2} .
$$

This completes the proof.

Remark 4.3. In general, the inequality (4.22) does not imply that $\left\{\Gamma_{x, j}: x \in \mathcal{X}, j \in\right.$ $J\}$ is a semi-continuous $g$-frame regardless how small the parameters $\lambda_{1}, \lambda_{2}, \mu$ are. A counterexample for $g$-frames can be found in [28], and an example can be constructed similarly for semi-continuous $g$-frames.

Corollary 4.4. Let $\left\{\Lambda_{x, j}: x \in \mathcal{X}, j \in J\right\}$ be a semi-continuous g-frame for $\mathcal{H}$ with respect to $(\mathcal{X}, \mu)$, with frame bounds $A, B$, and let $\left\{\Gamma_{x, j}: x \in \mathcal{X}, j \in J\right\}$ be a family in $\mathcal{L}\left(\mathcal{H}, \mathcal{K}_{x, j}\right)$ for any $x \in \mathcal{X}, j \in J$. Assume that there exists a constant $0<M<A$ such that

$$
\int_{\mathcal{X}} \sum_{j \in J}\left\|\left(\Lambda_{x, j}-\Gamma_{x, j}\right) f\right\|^{2} d \mu(x) \leq M\|f\|^{2}, \forall f \in \mathcal{H},
$$

then $\left\{\Gamma_{x, j}: x \in \mathcal{X}, j \in J\right\}$ is a semi-continuous g-frame for $\mathcal{H}$ with respect to $(\mathcal{X}, \mu)$, with bounds $A\left[1-(M / A)^{1 / 2}\right]^{2}$ and $B\left[1+(M / B)^{1 / 2}\right]^{2}$.

Proof. Let $\lambda_{1}=\lambda_{2}=0$ and $\mu=\sqrt{M}$. Since $M<A, \mu / \sqrt{A}=\sqrt{M / A}<1$. So, by Theorem 4.2, $\left\{\Gamma_{x, j}: x \in \mathcal{X}, j \in J\right\}$ is a semi-continuous $g$-frame for $\mathcal{H}$ with respect to $(\mathcal{X}, \mu)$, with bounds $A\left[1-(M / A)^{1 / 2}\right]^{2}$ and $B\left[1+(M / B)^{1 / 2}\right]^{2}$.

\section{Acknowledgments}

The author is deeply indebted to Dr. Azita Mayeli for several valuable comments and suggestions. The author is grateful to the United States-India Educational Foundation for providing the Fulbright-Nehru Doctoral Research Fellowship, and Department of Mathematics and Computer Science, the Graduate Center, City University of New York, New York, USA for its kind hospitality during the period of this work. He would also like to express his gratitude to the Norbert Wiener Center for Harmonic Analysis and Applications at the University of Maryland, College Park for its kind support.

\section{REFERENCES}

[1] S.T. Ali, J.P. Antoine, and J.P. Gazeau. Continuous frames in Hilbert spaces. Ann. Phys., 222(1):137, 1993.

[2] S.T. Ali, J.P. Antoine, and J.P. Gazeau. Coherent States, Wavelets and Their Generalizations. Springer-Verlag, New York, 2000.

[3] A. Askari-Hemmat, M.A. Dehghan, and M. Radjabalipour. Generalized frames and their redundancy. Proc. Amer. Math. Soc., 129(4):1143-1147, 2001. 
[4] R. Balan, P.G. Casazza, and D. Edidin. On signal reconstruction without phase. Appl. Comput. Harmon. Anal., 20(3):345-356, 2006.

[5] R. Balan, P.G. Casazza, D. Edidin, and G. Kutyniok. A new identity for Parseval frames. Proc. Amer. Math. Soc., 135(4):1007-1015, 2007.

[6] P.G. Casazza and O. Christensen. Perturbation of operators and applications to frame theory. $J$. Fourier Anal. Appl., 3(5):543-557, 1997.

[7] P.G. Casazza and G. Kutyniok. Frames of subspaces. Contemp. Math., 345:87-114, 2004.

[8] O. Christensen. An Introduction to Frames and Riesz Bases. Birkhäuser, Boston, 2003.

[9] O. Christensen and Y.C. Eldar. Oblique dual frames and shift-invariant spaces. Appl. Comput. Harmon. Anal., 17(1):48-68, 2004.

[10] B. Currey, A. Mayeli and V. Oussa. Characterization of shift-invariant spaces on a class of nilpotent Lie groups with applications. J. Fourier Anal. Appl., 20:384-400, 2014.

[11] I. Daubechies, A. Grossmann, and Y. Meyer. Painless nonorthogonal expansions. J. Math. Phys., 27(5):1271-1283, 1986.

[12] M.A. Dehghan and M.A. Hasankhani Fard. G-continuous frames and coorbit spaces. Acta Math. Acad. Paedagog. Nyházi (NS), 24:373-383, 2008.

[13] R.J. Duffin and A.C. Schaeffer. A class of nonharmonic Fourier series. Trans. Amer. Math. Soc., $72(2): 341-366,1952$.

[14] G.B. Folland. A Course in Abstract Harmonic Analysis. CRC Press, Boca Raton, Florida, 1995.

[15] M. Foranasier. Quasi-orthogonal decompositions of structured frames. J. Math. Anal. Appl., 289(1):180-199, 2004.

[16] M. Foranasier and H. Rauhut. Continuous frames, function spaces, and the discretization problem. J. Fourier Anal. Appl., 11(3):245-287, 2005.

[17] J.P. Gabardo and D. Han. Frames associated with measurable spaces. Adv. Comput. Math., 18(3):127-147, 2003.

[18] P. Găvruţa. On some identities and inequalities for frames in Hilbert spaces. J. Math. Anal. Appl., 321(1):469-478, 2006.

[19] L. Găvruţa. Frames for operators. Appl. Comput. Harmon. Anal., 32(1):139-144, 2012.

[20] L. Găvruţa. Atomic decompositions for operators in reproducing kernel Hilbert spaces. Math. Reports., 17(67)3:303-314, 2015.

[21] D. Geller. Fourier analysis on the Heisenberg group. Proc. Natl. Acad. Sci. U.S.A., 74(4):1328-1331, 1977.

[22] K. Gröchenig. Foundations of Time-Frequency Analysis. Birkhäuser, Boston, 2001.

[23] G. Kaiser. A Friendly Guide to Wavelets. Birkhäuser, Boston, 1994.

[24] S. Li and H. Ogawa. Pseudoframes for subspaces with applications. J. Fourier Anal. Appl., 10(4):409-431, 2004.

[25] A. Poria. Some identities and inequalities for Hilbert-Schmidt frames. Mediterr. J. Math., 14(2): Art. 59, 14 pp., 2017.

[26] A. Poria. Approximation of the inverse frame operator and stability of Hilbert-Schmidt frames. Mediterr. J. Math., 14(4): Art. 153, 22 pp., 2017.

[27] W. Sun. G-frames and g-Riesz bases. J. Math. Anal. Appl., 322(1):437-452, 2006.

[28] W. Sun. Stability of g-frames. J. Math. Anal. Appl., 326(2):858-868, 2007.

[29] R.M. Young. An Introduction to Non-Harmonic Fourier Series. Academic Press, New Work, 1980.

[30] X. Zhu and G. Wu. A note on some equalities for frames in Hilbert spaces. Appl. Math. Lett., 23(7):788-790, 2010.

Department of Mathematics, School of Engineering and Applied Sciences, Bennett University, Greater Noida, Uttar Pradesh, India

Department of Mathematics, Indian Institute of Science, Bengaluru, Karnataka, India

Anirudha Poria: anirudhap@iisc.ac.in

Received 13/05/2020 